\title{
Electrical Coupling and Excitatory Synaptic Transmission between Rhythmogenic Respiratory Neurons in the PreBötzinger Complex
}

\author{
Jens C. Rekling, Xuesi M. Shao, and Jack L. Feldman \\ Department of Neurobiology, University of California, Los Angeles, Los Angeles, California 90095-1763
}

\begin{abstract}
Breathing pattern is postulated to be generated by brainstem neurons. However, determination of the underlying cellular mechanisms, and in particular the synaptic interactions between respiratory neurons, has been difficult. Here we used dual recordings from two distinct populations of brainstem respiratory neurons, hypoglossal (XII) motoneurons, and rhythmogenic (type-1) neurons in the preBötzinger complex (preBötC), the hypothesized site for respiratory rhythm generation, to determine whether electrical and chemical transmission is present. Using an in vitro brainstem slice preparation from newborn mice, we found that intracellularly recorded pairs of XII motoneurons and pairs of preBötC inspiratory type- 1 neurons showed bidirectional electrical coupling. Coupling strength was
\end{abstract}

low $(<0.10)$, and the current that passed between two neurons was heavily filtered (corner frequency, $<10 \mathrm{~Hz}$ ). Dual recordings also demonstrated unidirectional excitatory chemical transmission (EPSPs of $\sim 3 \mathrm{mV}$ ) between type- 1 neurons. These data indicate that respiratory motor output from the brainstem involves gap junction-mediated current transfer between motoneurons. Furthermore, bidirectional electrical coupling and unidirectional excitatory chemical transmission are present between type-1 neurons in the preBötC and may be important for generation or modulation of breathing rhythm.

Key words: respiration; preBötzinger complex; type-1 neurons; respiratory rhythmogenesis; electrical coupling; excitatory transmission; newborn mice
Understanding the synaptic interactions among brainstem neurons is a prerequisite to understanding how breathing rhythm is generated and cranial respiratory motor output patterns are formed. We used an in vitro brainstem slice preparation from newborn mice (Smith et al., 1991; Rekling et al., 1996a) that retains the central network for respiratory rhythm generation to study synaptic transmission between neurons involved in generation of respiratory rhythm and motor output. The XII nucleus, containing respiratory-modulated XII motoneurons, and the preBötzinger complex (preBötC), containing rhythmogenic neurons (Rekling et al., 1996a; Rekling and Feldman, 1998; Gray et al., 1999), are encompassed within this slice, which spontaneously generates a respiratory-related rhythmic motor output in XII nerve rootlets. Two types of inspiratory neurons showing rhythmic discharges in phase with XII nerve activity are found in the preBötC (type-1 and type-2) (Rekling et al., 1996a; Rekling and Feldman, 1997). We have proposed that type-1 neurons are key neurons for rhythmogenesis because they are the first neurons to be active before the onset of inspiration, have intrinsic membrane properties including a long-lasting afterhyperpolarization that are rhythmogenic, and are the main targets in the preBötC for peptidergic input regulating respiratory frequency (Rekling et al., 1996a,b; Rekling and Feldman, 1998; Gray et al., 1999). In the group-pacemaker hypothesis (Rekling and Feldman, 1998), recurrent excitatory synaptic interactions between type-1 neurons underlie their rhythmogenic potential. In this study, we under-

\footnotetext{
Received May 30, 2000; revised Sept. 6, 2000; accepted Sept. 11, 2000.

This work was supported by National Institutes of Health Grants NS24742 and HL40959. J.C.R. was supported by the Parker B. Francis Foundation.

Correspondence should be addressed to Dr. Jack L. Feldman, Department of Neurobiology, Box 951763, University of California, Los Angeles, Los Angeles, CA 90095-1763. E-mail: feldman@ucla.edu.

Dr. Rekling's present address: H. Lundbeck A/S, Ottiliavej 9, 2500 Valby, Denmark. Copyright $(C) 2000$ Society for Neuroscience $\quad 0270-6474 / 00 / 200001-05 \$ 15.00 / 0$
}

took a series of dual recordings to determine whether direct recurrent synaptic interactions exist between type-1 neurons.

Chemical synaptic transmission is the dominant form of cellcell signaling in the nervous system, whereas electrical coupling between neurons has been viewed as a less important form of signaling, mainly used in phylogenetically older organisms. This view has been challenged by recent experiments suggesting that electrical coupling is involved in complex functions in mammalian CNS (Welsh et al., 1995; Draguhn et al., 1998; Bennett, 1999; Usher et al., 1999; Tresch and Kiehn, 2000). Given the importance of gap junctions in rhythmic brain activity (Velazquez and Carlen, 2000), the current experiments were designed so both chemical and electrical synaptic interactions between rhythmically active respiratory neurons could be detected.

\section{MATERIALS AND METHODS}

Neonate (days 1-3) BALB/c mice were deeply anesthetized by hypothermia and killed, and a 400- to 600- $\mu \mathrm{m}$-thick transverse slice of the brainstem was cut on a vibratome (Smith et al., 1991). The preparation was transferred to a $0.2 \mathrm{ml}$ recording chamber and was superfused (3 $\mathrm{ml} / \mathrm{min})$ with preheated $\left(28.0^{\circ} \mathrm{C}\right)$ oxygenated $\left(95 \% \mathrm{O}_{2}-5 \% \mathrm{CO}_{2}, \mathrm{pH} 7.4\right)$ artificial CSF (aCSF). The aCSF solution contained (in mM): $130 \mathrm{NaCl}$, $5.4 \mathrm{KCl}, 0.8 \mathrm{KH}_{2} \mathrm{PO}_{4}, 26 \mathrm{NaHCO}_{3}, 30$ glucose, $1 \mathrm{MgCl}_{2}$, and $0.8 \mathrm{CaCl}_{2}$.

This article is published in The Journal of Neuroscience, Rapid Communications Section, which publishes brief, peerreviewed papers online, not in print. Rapid Communications are posted online approximately one month earlier than they would appear if printed. They are listed in the Table of Contents of the next open issue of JNeurosci. Cite this article as: JNeurosci, 2000, 20:RC113 (1-5). The publication date is the date of posting online at www.jneurosci.org.

http://www.jneurosci.org/cgi/content/full/4745 
A suction electrode placed on an XII nerve root recorded rhythmic respiratory-related motor output. Nerve activity was amplified and bandpass filtered at $3 \mathrm{~Hz}$ to $1 \mathrm{kHz}$ by an amplifier. A HumBug (Quest Scientific, North Vancouver, Canada) eliminated $60 \mathrm{~Hz}$ noise, and rectified nerve activity was integrated ( $\mathrm{RC}$ circuit, time constant of 50 msec). Glass micropipettes (resistances typically 2-3 M $\Omega$ ) were filled with a solution containing (in $\mathrm{mM}$ ): $115 \mathrm{HMeSO}_{3}, 115 \mathrm{KOH}, 5 \mathrm{NaCl}, 1$ $\mathrm{MgCl}_{2}, 0.01 \mathrm{CaCl}_{2}, 0.1$ BAPTA, 10 HEPES, and 3 2-ATP $\left(\mathrm{Mg}^{2+}\right), \mathrm{pH}$ 7.3. Neurons were visualized using differential interference contrast and infrared video microscopy, and simultaneous whole-cell patch-clamp recordings (current-clamp mode) from pairs of XII motoneurons and type-1 neurons in the preBötC were done using two Axoclamp-2A (Axon Instruments, Foster City, CA) amplifiers. XII motoneurons were identified by their distinct anatomical location, and type- 1 neurons were identified by their location in the preBötC and their characteristic electroresponsive properties (Rekling et al., 1996a; Gray et al., 1999). Signals were recorded on videocassette (pulse code modulation; Vetter Instruments), digitized at $1-20 \mathrm{kHz}$ using a Digidata 1200 analog-to-digital board (Axon Instruments), and analyzed on a personal computer using Clampex 7 software (Axon Instruments). The coupling coefficient between two electrically coupled neurons was calculated as the ratio of membrane potential deflections $\left(\Delta V_{2} / \Delta V_{1}\right)$ in response to a $200 \mathrm{pA}, 1 \mathrm{sec}$ hyperpolarizing pulse. Prejunctional and postjunctional potential shapes were determined by spike-triggered averaging of $\sim 50$ prejunctional action potentials elicited by $200 \mathrm{pA}, 1 \mathrm{sec}$ depolarizing pulses. Action potential duration was measured at the inflection point of the spike upstroke. A small, fast $(\sim 0.2 \mathrm{mV}, \sim 3 \mathrm{msec})$ biphasic spike artifact was noted also in dual intracellular-to-extracellular recordings between uncoupled XII motoneurons $(n=3)$ (see Fig. $3 B)$ and probably reflect cross-talk between the two Axoclamp-2A head stages.

The ZAP current input with a constant amplitude and a linearly decreasing (or increasing) frequency (Puil et al., 1986) was generated digitally from the equation: $I(x)=2 \sin \left(400 * x^{2}\right)$, for $x=1$ to 0 in 4096 steps. At $1 \mathrm{kHz}$ digitizing frequency, this gave a waveform with frequency range of 0 to $\sim 30 \mathrm{~Hz}$. Voltage and current traces were transformed into the frequency domain using a Fast Fourier Transform (FFT) (rectangular window method). The results were smoothed (10 point adjacent averaging), and impedance-magnitude plots were generated by plotting normalized $\operatorname{FFT}(V) / \mathrm{FFT}(I)$ versus frequency. Corner frequency of these plot were defined as attenuation to $-3 \mathrm{~dB}=1 / \sqrt{ } 2$ of lowest frequency response (boundary frequencies were inherently noisy, and the first $1 \mathrm{~Hz}$ of the range was ignored). There was no difference in FFT plots comparing injections of ZAP functions with decreasing or increasing frequencies in the time domain. FFT analysis on burst of inspiratory EPSPs were done on smoothed traces (100 point adjacent averaging, 10 $\mathrm{kHz}$ sampling rate), and peak and baseline values were determined from the resulting amplitude plots. EPSP amplitude, rise time (10-90\%), decay time constant (single exponential fit), and synaptic delay (from peak of presynaptic spike to onset of postsynaptic EPSP) in pairs of type-1 neurons showing chemical transmission were measured on averaged traces ( $>5$ sweep, discarding failures) at a hyperpolarized postsynaptic membrane potential. In paired recordings, the term "cell 1" refers to the neuron receiving a current injection (electrically coupled pair) or the presynaptic neuron (chemical transmission). TTX (1 $\mu \mathrm{M}$; Sigma, St. Louis, MO) and $\mathrm{CdSO}_{4}(100 \mu \mathrm{M}$; Sigma) was applied to the bath in some experiments. Statistics are given as mean $\pm \mathrm{SD}$, unless otherwise stated.

\section{RESULTS}

We made simultaneous whole-cell patch-clamp recordings from pairs of XII motoneurons and from pairs of type-1 neurons in the preBötC.

\section{Electrical coupling between inspiratory-modulated XII motoneurons}

Evidence for bidirectional electrical coupling was seen in 9 of 36 $(25 \%)$ dual recordings from inspiratory-modulated X II motoneurons, i.e., the neurons had bursts of EPSPs in phase with activity on the XII nerve (Fig. 1D). The distance between pipette tips in recorded coupled XII motoneuron pairs was $50 \pm 8 \mu \mathrm{m}$ (range, 34-66 $\mu \mathrm{m} ; n=9)$; this compares with a maximum motoneuron diameter of $19 \pm 3 \mu \mathrm{m}$ (range, 13-24 $\mu \mathrm{m} ; n=20$ ). After blockade of action potential and $\mathrm{Ca}^{2+}$-dependent synaptic transmission
A
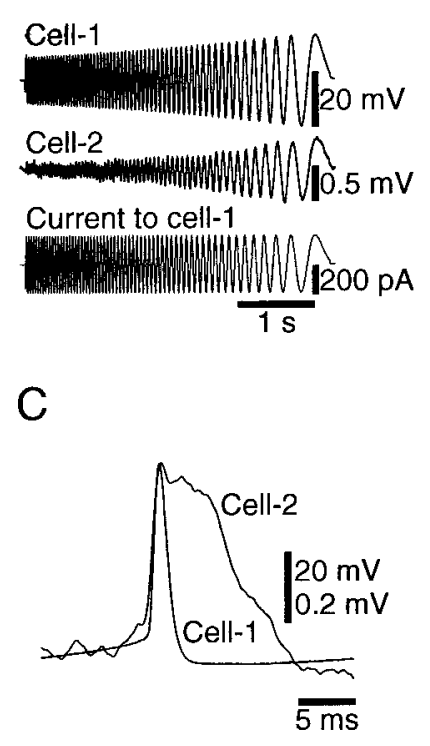

B
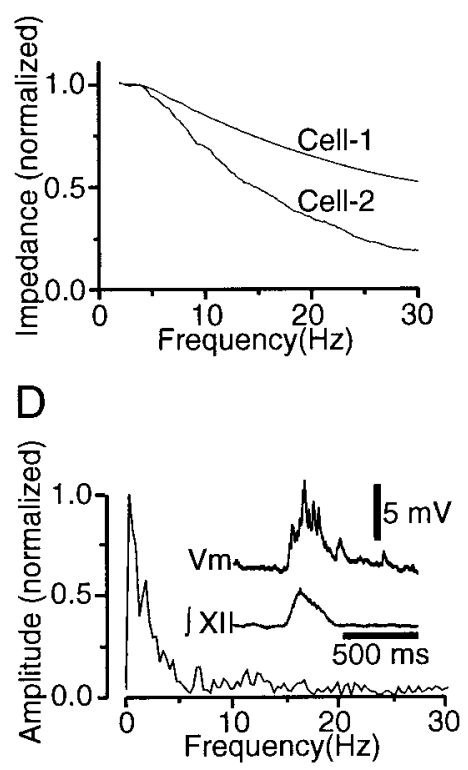

Figure 1. Respiratory-modulated XII motoneurons are electrically coupled. $A$, Simultaneous recordings from two XII motoneurons. A ZAP current waveform injected into cell 1 elicited an attenuated and filtered response in the electrically coupled cell $2\left(1 \mu \mathrm{M}\right.$ TTX and $100 \mu \mathrm{M} \mathrm{Cd}^{2+}$ were added to the bathing solution). $B$, Impedance magnitude versus frequency plot of one neuron pair based on FFT analysis of the membrane responses to the ZAP current input in cell 1 and cell 2 . The corner frequency $(-3 \mathrm{~dB})$ of cell 1 was $17 \mathrm{~Hz}$, whereas cell 2 was $9 \mathrm{~Hz} . C$, Superposition of an action potential in cell 1 that produced a distorted waveform in cell 2. $D$, Plot of the frequency components contained in a burst of inspiratory EPSPs $\left(V_{\mathrm{m}}\right.$, inset $)$ in an XII motoneuron.

(by adding TTX and $\mathrm{Cd}^{2+}$ to the superfusing solution), hyperpolarizing or depolarizing current injections into one motoneuron gave rise to attenuated membrane responses in an electrically coupled motoneuron (data not shown); the coupling coefficient ranged between 0.008 and $0.077(n=4$ pairs). We then injected a ZAP current waveform into either of the motoneurons (Fig. $1 A$ ), and an attenuated waveform in the postjunctional motoneuron with low-pass filter characteristics was produced (Fig. 1A). Prejunctional motoneurons filtered the ZAP current input with a corner frequency $(-3 \mathrm{~dB})$ of $19 \pm 5 \mathrm{~Hz}$, whereas postjunctional motoneurons filtered the transferred current with a lower corner frequency of $7 \pm 4 \mathrm{~Hz}(n=4)$ (Fig. $1 B)$. The envelope of the inspiratory burst of EPSPs in XII motoneurons was composed mainly of frequencies $\sim 5 \mathrm{~Hz}$ and below, with a peak at $1.0 \pm 0.3$ $\mathrm{Hz}(n=11)$ (Fig. $1 D)$.

This marked frequency-dependent attenuation of signals traveling between electrically coupled motoneurons gave rise to severely distorted action potential waveforms in postjunctional motoneurons (Fig. 1C). The duration of the prejunctional action potentials was $3.5 \pm 1.0 \mathrm{msec}$, and the postjunctional potential was longer lasting at $15.4 \pm 3.8 \mathrm{msec}$.

\section{Electrical coupling between rhythmogenic type-1 neurons}

Three of 23 pairs (13\%) of type-1 neurons were bidirectionally electrically coupled (Fig. $2 A$ ). The coupling coefficient ranged between 0.009 and 0.11 ( $n=3$ pairs). The maximal somatic diameter of type- 1 neurons was $15.3 \pm 1.4 \mu \mathrm{m}$ (range, $13-17 \mu \mathrm{m}$; $n=10$ ), and the distance between pipette tips in type- 1 pairs that were electrotonically coupled was 52 and $66 \mu \mathrm{m}(n=2$, one pair 
A

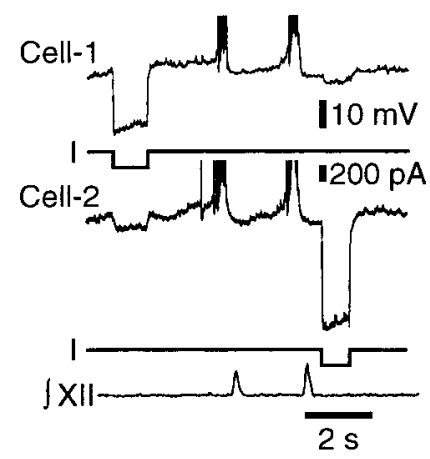

B

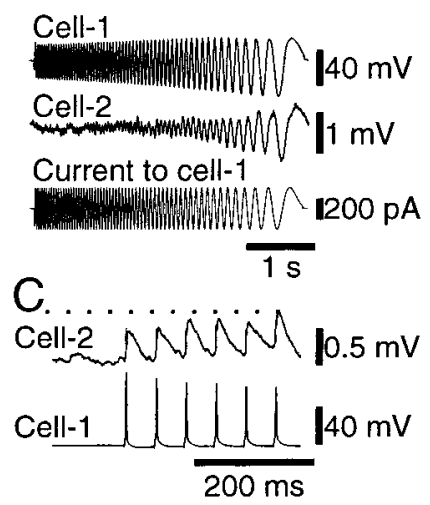

D

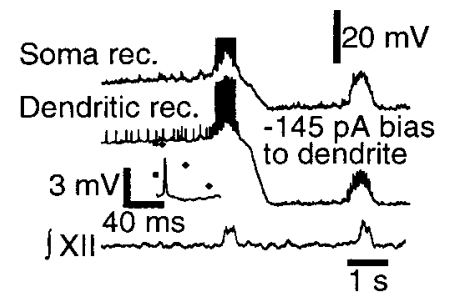

E

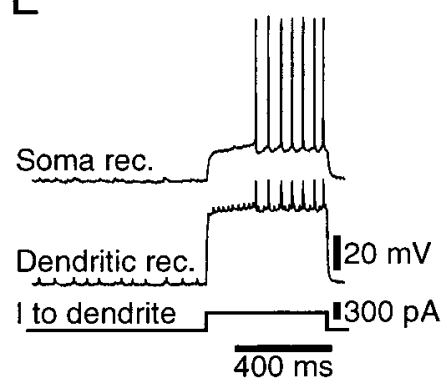

Figure 2. Type-1 neurons are bidirectionally electrically coupled. $A$, Simultaneous recordings from two type-1 neurons (spikes are truncated). A hyperpolarizing pulse in either neuron elicited an attenuated hyperpolarizing responses in the other electrically coupled neuron. $B$, ZAP current injection into cell 1 elicited an attenuated and filtered response in the coupled cell 2. $C$, Train of prejunctional action potentials in cell 1 and corresponding temporally summated (dotted line) postjunctional coupling potential waveforms in a coupled cell 2. $D$, Soma-dendritic dual recording from a type-1 neuron, with spikelets in the dendritic, but not in the somatic, recording. $E$, Current injection into the dendrite increases the frequency of spikelets.

not measured). Prejunctional type-1 neurons filtered a ZAP current input with a corner frequency of $39 \pm 17 \mathrm{~Hz}$, whereas postjunctional neurons filtered the transferred current with a lower corner frequency of $9 \pm 5 \mathrm{~Hz}(n=3$ pairs) (Fig. $2 B)$. The envelope of the inspiratory burst of EPSPs in type- 1 neurons was composed mainly of frequencies below $\sim 7 \mathrm{~Hz}$, with a peak at $1.0 \pm 0.4 \mathrm{~Hz}(n=3)$.

The duration of prejunctional action potentials was $3.1 \pm 1.3$ msec, and the postjunctional potentials were longer lasting (19.9 \pm $3.9 \mathrm{msec} ; n=3$ pairs). Postjunctional coupling potential waveforms resembled small-amplitude EPSPs (without any measurable synaptic delay) and displayed temporal summation (Fig. 2C).

The somas of coupled type-1 neurons did not touch each other. Thus, gap junctions linking pairs of type-1 neurons appear dendro-dendritic or dendro-somatic. Direct support for this notion comes from one accidental soma-dendritic dual recording (40 $\mu \mathrm{m}$ between pipettes) from a single type-1 neuron. Spikelets ( 3 $\mathrm{mV}$ amplitude, action potential shape) presumably generated in a second electrical coupled inspiratory neuron were seen in the dendritic, but not in the somatic, recording (Fig. 2D). Steady hyperpolarizing current or depolarizing pulses injected into the dendrite reduced or increased the frequency of the spikelets, respectively (Fig. $2 D, E$ ), suggesting that the injected current passed through dendritic gap junctions, changing the membrane potential and firing frequency of the coupled neuron.

A
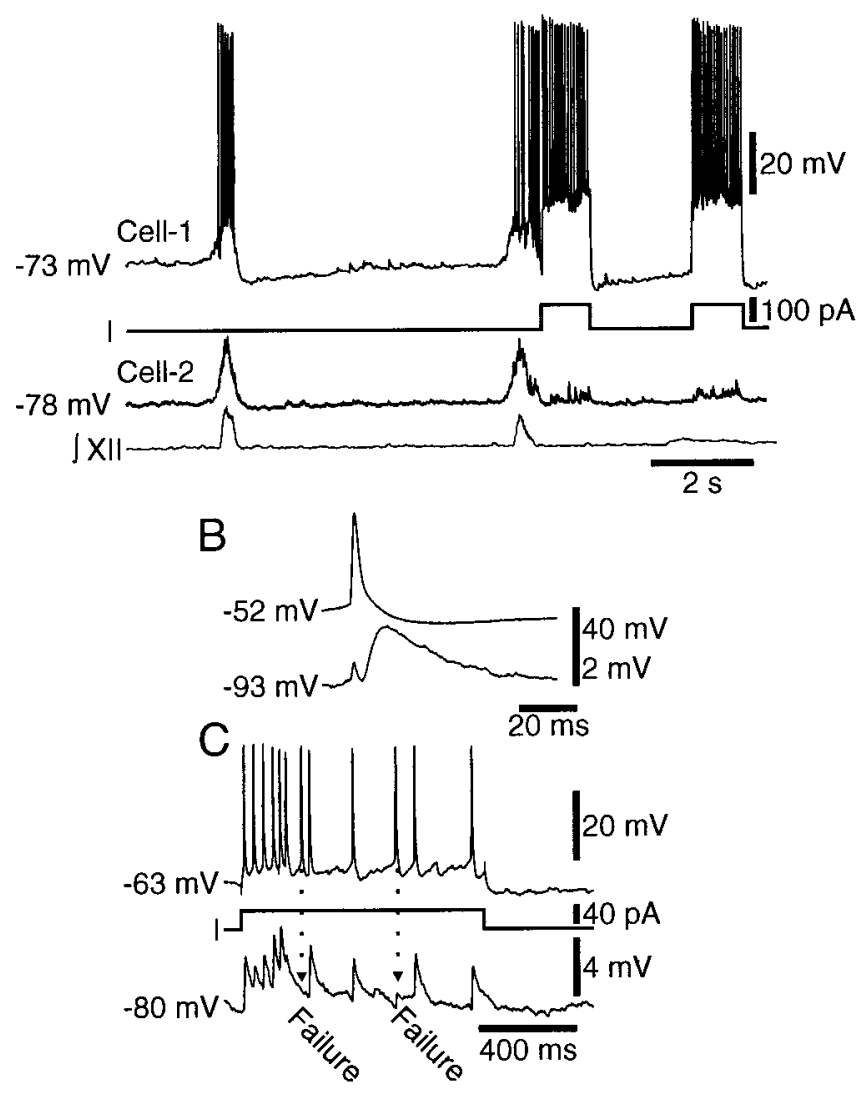

Figure 3. Type-1 neurons show unidirectional excitatory chemical transmission to other type- 1 neurons. $A$, Simultaneous recordings from two type-1 neurons, one with no injected current (top trace) and the other at a hyperpolarized membrane potential ( $-72 \mathrm{pA}$ bias current). Transient depolarizing current injection into cell 1 elicited repetitive firing in that cell and trains of EPSPs in cell 2. B, Presynaptic action potential (top trace) and postsynaptic EPSP waveforms (bottom trace). Note that the small potential preceding the EPSP is likely head stage-to-head stage cross-talk (see Materials and Methods). $C$, Current pulse to cell 1 elicited repetitive firing and EPSPs with intermittent synaptic transmission failures in cell 2 .

Nonrhythmogenic type-2 neurons are the second type of inspiratory neurons found in the preBötC (Rekling et al., 1996a). Although no electrical (or chemical) coupling was found between type- 1 and type- 2 neurons ( $n=9$ pairs), we cannot exclude its existence.

\section{Pharmacological blockade of gap junctions is inconclusive}

We bath applied or local perfused over the preBötC four different gap junction blockers (heptanol, $1 \mathrm{~mm}$; octanol, $1 \mathrm{~mm}$; carbenoxolone, $100 \mu \mathrm{M}$; and halothane, $10 \mathrm{~mm}$ ), which all reversibly suppressed the rhythmic output on the XII nerve. However, all of these compounds had substantial adverse effects on the membrane excitability of both XII motoneurons and type-1 neurons. Before and after applying these drugs, we current clamped a neuron at $-60 \mathrm{mV}$, applied a series of current pulses $(500 \mathrm{msec}$; -110 to $+170 \mathrm{pA} ; 20 \mathrm{pA}$ increments), and measured the number of action potentials when the current pulse depolarized the neuron to approximately $-50 \mathrm{mV}$. Heptanol greatly reduced the number of action potentials in XII motoneurons without changing input resistance (control, $13.3 \pm 0.4$ spikes $/ 500 \mathrm{msec}$; heptanol, $1 \pm 1$ spikes $/ 500 \mathrm{msec} ; n=2$ ). Carbenoxolone suppressed 
and finally eliminated respiratory-related output after 45-60 min. Input resistance of type- 1 preBötC neurons $(n=4)$ was reduced from $323 \pm 62$ to $176 \pm 37 \mathrm{M} \Omega$ at $20-30 \mathrm{~min}$ in carbenoxolone containing aCSF and further reduced to $151 \pm 10 \mathrm{M} \Omega$ at $>45$ min. Carbenoxolone also reduced the number of action potentials elicited by depolarizing current pulses from $11.9 \pm 3.5$ to $8.4 \pm$ 5.0 spikes $/ 500 \mathrm{msec}$ at $20-30 \mathrm{~min}$ and further reduced firing to 0 at $>45$ min. (at which point, action potentials could not be generated by further depolarizing the neuron). These effects of heptanol and carbenoxolone were partially reversible after washout with fresh aCSF.

\section{Excitatory chemical transmission between type-1 neurons}

Three of 23 pairs (13\%) of type-1 neurons were chemically synaptically coupled (Fig. $3 A$ ), showing unidirectional EPSPs, i.e., synaptic potentials in only one direction. The EPSPs had a synaptic delay of $1.3 \pm 1.1 \mathrm{msec}$, amplitude of $2.8 \pm 1.5 \mathrm{mV}$, rise time of $2.5 \pm 1.4 \mathrm{msec}$, and decay time constant of $21.3 \pm 5.6 \mathrm{msec}$ $(n=3)$ (Fig. 3B). Synaptic transmission was not $100 \%$ efficient, because there were some failures (Fig. 3C). We did not measure the probability of failures, because we were unable to obtain sufficiently long-term recordings for such analysis. None of the pairs of chemically coupled type- 1 neurons showed evidence of electrical coupling.

\section{DISCUSSION}

This study demonstrates that electrical coupling is present between respiratory-modulated XII motoneurons and that both electrical and excitatory chemical transmission is present between type-1 neurons.

Electrical coupling between respiratory-modulated XII motoneurons is consistent with the observation that genioglossal motoneurons show dye-coupling (Mazza et al., 1992). Inspiratory-modulated motoneurons in nucleus ambiguus (Rekling and Feldman, 1997) also show electrical coupling. Electrical coupling among perinatal rat phrenic motoneurons might be involved in synchronizing respiratory drive to the diaphragm (MartinCaraballo and Greer, 1999). Electrical coupling between spinal motoneurons (Walton and Navarrete, 1991) underlies action potential-independent coordination of spinal motor rhythms (Tresch and Kiehn, 2000), and similar coordination of brainstem respiratory motoneurons may facilitate coordination of upper airway motor patterns.

Electrical coupling between rhythmogenic type- 1 neurons is likely to affect respiratory rhythm generation because, in computational models of interacting neurons, electrical coupling is rhythm promoting (Sherman and Rinzel, 1992; Traub, 1995; Moortgat et al., 2000). Respiratory rhythm generation may also involve action potential-independent oscillations mediated by gap junctions, because oscillations in the absence of action potentials are present in spinal motor systems (Tresch and Kiehn, 2000). Bidirectional electrical coupling between type-1 neurons could act, in concert with intrinsic conductances, to synchronize and amplify oscillatory respiratory activity at slow time scales. Interference with breathing rhythm after pharmacological blockade of gap junctions would support such a role for electrical coupling in respiratory rhythmogenesis. All of these compounds had substantial adverse effects on the membrane excitability of both XII motoneurons and type- 1 neurons. Thus, direct evidence for a possible role of gap junctions in respiratory rhythm generation awaits development of gap junction blockers that do not otherwise affect neuronal excitability.

We cannot exclude the possibility that electrical coupling is present between type- 1 neurons only in newborn mice, but the neuron-specific connexin 36 (Cx36) (Condorelli et al., 1998) is seen in the preBötC in adult mice (Parenti et al., 2000), and Cx26 and $\mathrm{Cx} 32$ are seen in presumptive type- 1 neurons in adult rats (O'Neal et al., 2000). This suggests that gap junctions persist in this region in adult rodents. Models of electrical coupling predict that coupling strength can affect the frequency of oscillatory networks (Kepler et al., 1990; Moortgat et al., 2000). Thus, if electrical coupling between type-1 neurons is modifiable, e.g., via phosphorylation of connexins, breathing frequency could be modulated by the regulation of coupling strength.

The results of dual recordings provide us a basis to estimate the number of directly connected neurons (see Appendix). We have estimated that there are up to 300 type- 1 neurons in each preBötC; these neurons have dense intermingled dendrites (P. A. Gray and J. L. Feldman, unpublished observations). Given the compact size of the preBötC (Gray et al., 1999), especially compared with the dendritic lengths of type- 1 neurons $(>1.5 \mathrm{~mm})$, we assume that the likelihood of coupling is not distant-dependent. Thus, if there are 100 neurons, given that we found 3 of 23 pairs of type-1 neurons showing electrical couplings, every type- 1 neuron would be connected to $\sim 13$ other type- 1 neurons (Eq. 1 in Appendix). This estimate $(1 \Rightarrow 13)$ is between the estimate of dye coupling using Lucifer yellow (Connors et al., 1983) and the estimate using Neurobiotin in rat neocortical neurons (Peinado et al., 1993; Rörig et al., 1995). If there are as many as 300 type-1 neurons, then the estimate is $\sim 1 \Rightarrow 39$ connections.

There was strong filtering of signals traveling between electrically coupled pairs of XII motoneurons and of type- 1 neurons, which was minimal at frequencies with the most power in the inspiratory synaptic drive $(\sim 5 \mathrm{~Hz})$. We propose that this coupling synchronizes their membrane potential at frequencies corresponding to the envelope of the inspiratory EPSP burst. Furthermore, we hypothesize that regenerative membrane currents (somatic action potentials, dendritic $\mathrm{Ca}^{2+}$ currents) elicited by the inspiratory synaptic drive pass through gap junctions, adding a low-pass filtered current to the inspiratory synaptic drive in the coupled neurons. In this manner, electrical coupling amplifies and synchronizes type-1 neuron depolarization, predicted to underlie respiratory rhythm generation, as well as the respiratory motor output from XII motoneurons.

Excitatory chemical transmission between type- 1 neurons is in agreement with recent experiments showing synchronization, mediated by glutamatergic synaptic interactions, between endogenous bursting neurons in the preBötC (Koshiya and Smith, 1999). Type-1 neurons may be identical to or overlap as a group with these neurons, which show intrinsic oscillatory bursting properties under elevated extracellular potassium concentrations (Smith et al., 1991; Koshiya and Smith, 1999), because 50\% of type-1 neurons have bursting properties (Rekling et al., 1996a). In our small sample of dual recordings, we found that chemically coupled pairs of type-1 neurons were not electrically coupled (and visa versa), which suggests that an exclusive electricalchemical network rule is implemented in synaptic connections between type- 1 neurons. In keeping with the group-pacemaker hypothesis (Rekling and Feldman, 1998), excitatory chemical transmission may be the driving force for generation of the inspiratory burst in type-1 neurons, whereas electrical coupling 
may phase-lock the membrane potential during both inspiration and expiration in these rhythmogenic neurons.

\section{Appendix}

\section{Estimation of number of connected neurons based on dual recordings}

Suppose we have $N$ neurons, and their interconnections are homogenous. There are $W=N(N-1) / 2$ pairs of neurons. If we do $n$ dual recordings and get $b$ pairs of coupled neurons, the estimated rate of coupling is $\hat{\theta}=b / n$. The estimated total number of pairwise couplings among $N$ neurons would be $\mathrm{W} * \hat{\theta}$. Because each connection is shared by two neurons, every neuron is connected (on average) to $m$ other neurons, where $m=\mathrm{W} * \hat{\theta} * 2 / N$. By substitution,

$$
m=\frac{N(N-1)}{2} \frac{\hat{\theta}}{N} * 2=(N-1) \hat{\theta}
$$

Equation 1 can be derived in another way. If every neuron is connected to other $m$ neurons and if we patch onto one neuron, the probability of a second electrode patching onto a connected neuron would be $\theta=m /(N-1)$.

The SD of the estimate $\hat{\theta}$ is given by (Rice, 1988)

$$
\operatorname{SD}(\hat{\theta})=\sqrt{\frac{\hat{\theta}(1-\hat{\theta})}{n-1}}
$$

\section{REFERENCES}

Bennett MVL (2000) Seeing is relieving: electrical synapses between visualized neurons. Nat Neurosci 3:7-9.

Condorelli D, Parenti R, Spinella F, Salinaro A, Belluardo N, Cardile V, Cicirata F (1998) Cloning of a new gap junction gene (Cx36) highly expressed in mammalian brain neurons. Eur J Neurosci 10:1202-1208. Connors BW, Benardo LS, Prince DA (1983) Coupling between neurons of the developing rat neocortex. J Neurosci 3:773-782.

Draguhn A, Traub RD, Schmitz D, Jefferys JG (1998) Electrical coupling underlies high-frequency oscillations in the hippocampus in vitro. Nature 394:189-192.

Gray PA, Rekling JC, Bocchiaro CM, Feldman JL (1999) Modulation of respiratory frequency by peptidergic input to rhythmogenic neurons in the preBötzinger complex. Science 286:1566-1568.

Kepler TB, Marder E, Abbott LF (1990) The effect of electrical coupling on the frequency of model neuronal oscillators. Science 248:83-85.

Koshiya N, Smith JC (1999) Neuronal pacemaker for breathing visualized in vitro. Nature 400:360-363.

Martin-Caraballo M, Greer JJ (1999). Electrophysiological properties of rat phrenic motoneurons during perinatal development. J Neurophysiol 81:1365-1378.
Mazza E, Nunez AP, Spielmann J, Cameron W (1992) Anatomical and electrotonic coupling in developing genioglossal motoneurons of the rat. Brain Res 598:127-137.

Moortgat KT, Bullock TH, Sejnowski TJ (2000) Gap junction effects on precision and frequency of a model pacemaker network. J Neurophysiol 83:984-997.

O'Neal MH, Halat TJ, Edelman NH, Solomon IC (2000) Co-expression of gap junction proteins with $\mu$-opioid and substance p receptors in the pre-Bötzinger complex of neonatal and adult rats. Soc Neurosci Abstr 26:927.

Parenti R, Gulisano M, Zappala A, Cicirata F (2000) Expression of connexin36 mRNA in adult rodent brain. NeuroReport 11:1497-1502.

Peinado A, Yuste R, Katz LC (1993) Extensive dye coupling between rat neocortical neurons during the period of circuit formation. Neuron 10:103-114.

Puil E, Gimbarzevsky B, Miura RM (1986) Quantification of membrane properties of trigeminal root ganglion neurons in guinea pigs. J Neurophysiol 55:995-1016.

Rekling JC, Feldman JL (1997) Bidirectional electrical coupling between inspiratory motoneurons in the newborn mouse nucleus ambiguus. J Neurophysiol 78:3508-3510.

Rekling JC, Feldman JL (1998) PreBötzinger complex and pacemaker neurons: hypothesized site and kernel for respiratory rhythm generation. Annu Rev Physiol 60:385-405.

Rekling JC, Champagnat J, Denavit-Saubié M (1996a) Electroresponsive properties and membrane potential trajectories of three types of inspiratory neurons in the newborn mouse brain stem in vitro. J Neurophysiol 75:795-810.

Rekling JC, Champagnat J, Denavit-Saubié M (1996b) Thyrotropinreleasing hormone (TRH) depolarizes a subset of inspiratory neurons in the newborn mouse brain stem in vitro. J Neurophysiol 75:811-819.

Rice JA (1988) Survey sampling. In: Mathematical statistics and data analysis, pp175-213. Belmont, CA: Wadsworth.

Rörig B, Klausa G, Sutor B (1995) Dye coupling between pyramidal neurons in developing rat prefrontal and frontal cortex is reduced by protein kinase A activation and dopamine. J Neurosci 15:7386-400.

Sherman A, Rinzel J (1992) Rhythmogenic effects of weak electrotonic coupling in neuronal models. Proc Natl Acad Sci USA 89:2471-2474.

Smith JC, Ellenberger HH, Ballanyi K, Richter DW, Feldman JL (1991) Pre-Bötzinger complex: a brainstem region that may generate respiratory rhythm in mammals. Science 254:726-729.

Traub RD (1995) Model of synchronized population bursts in electrically coupled interneurons containing active dendritic conductances. J Comput Neurosci 2:283-289.

Tresch MC, Kiehn O (2000) Motor coordination without action potentials in the mammalian spinal cord. Nat Neurosci 3:593-599.

Usher M, Cohen JD, Servan-Schreiber D, Rajkowski J, Aston-Jones G (1999) The role of locus coeruleus in the regulation of cognitive performance. Science 283:549-554.

Velazquez JLP, Carlen PL (2000) Gap junctions, synchrony and seizures. Trends Neurosci 23:68-74.

Walton K, Navarrete R (1991) Postnatal changes in motoneurone electrotonic coupling studied in the in vitro rat lumbar spinal cord. J Physiol (Lond) 433:283-305.

Welsh JP, Lang EJ, Suglhara I, Llinás R (1995) Dynamic organization of motor control within the olivocerebellar system. Nature 374:453457. 